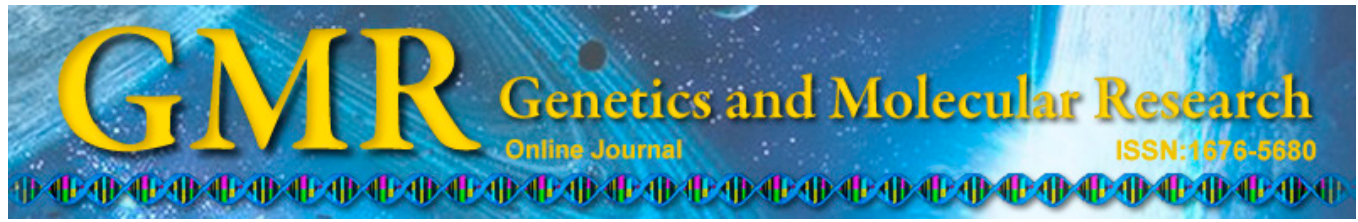

\title{
Influence of thoracic duct ligation on the lipid metabolism of patients with esophageal carcinoma after esophagectomy
}

\author{
J.P. Liu, Y.H. Zhang, B. Yang, Q. Chen and L. Cao \\ Department of Cardiothoracic Surgery, Suining Central Hospital, Sichuan, China \\ Corresponding author: Y.H. Zhang \\ E-mail:735013350@qq.com
}

Genet. Mol. Res. 14 (1): 2527-2536 (2015)

Received February 17, 2014

Accepted July 17, 2014

Published March 30, 2015

DOI http://dx.doi.org/10.4238/2015.March.30.11

\begin{abstract}
The aim of this study was to determine the influence of thoracic duct ligation on the lipid metabolism of patients with esophageal carcinoma after esophagectomy. A total of 74 patients with esophageal carcinoma who underwent esophagectomy were divided into 2 groups according to whether or not their thoracic duct was ligated. Thirty-nine patients were in the thoracic duct ligation group and the other 35 assigned to the control group. Enteral feeding was through a nasojejunal tube from the 1 st day to the 8 th day after operation, and liquid diet was provided starting on the 6th day. We compared the plasma concentrations of cholesterol (CHOL), triglycerides (TG), high-density lipoprotein (HDL), and low-density lipoprotein (LDL) at different time points. There were no statistically significant differences between the two groups in CHOL, TG and HDL levels at different times. However, LDL levels in the thoracic duct ligation group were significantly lower at different times compared to the other group ( $\mathrm{P}$ $<0.05$ ), where they were the lowest at the end of the 1 st month and then gradually recovered 3 months later. Thoracic duct ligation can effectively prevent chylomicrons from being transferred to the blood,
\end{abstract}


reducing the generation of LDL. The establishment of collateral circulation was slow after the ligation of the thoracic duct, which had a negative effect on early postoperative nutrition of patients.

Key words: Thoracic duct; Esophageal cancer; Plasma lipid; Nutrition; Collateral circulation

\section{INTRODUCTION}

Esophageal carcinoma is one of the most common tumors threatening the health of people in China, and radical esophagectomy is the most effective treatment. Chylothorax is the most severe complication associated with esophagectomy, and the incidence is 0.4-4\% (Sauvanet, 2002; Cerfolio, 2006; Nair et al., 2007). The mortality is as high as $50 \%$ in some reports (Fahimi et al., 2001) due to its great harm and treatment difficulties. Therefore, it is necessary to prevent chylothorax, although it is difficult in esophageal carcinoma surgery. In recent years, many clinical studies in China and abroad have highlighted the importance and effectiveness of thoracic duct ligation in the prevention of chylothorax in patients undergoing esophageal carcinoma surgery (Cagol et al., 2009; Sokouti and Montazeri, 2009).

Few studies have discussed the influence of thoracic duct blockage on nutrition in patients who have undergone esophageal cancer surgery, and for enteral and parenteral nutrition, there are few definite answers about which one should be chosen first for such patients. Aiko et al. (2003) found that after blockage of lymphatic flow in the thoracic duct, a lymphatic collateral pathway gradually develops, and patients whose thoracic duct was ligated did not get any other benefit from early enteral nutrition. These authors then recommended that early enteral feeding is not suitable for patients whose thoracic duct is ligated, but the reason was not clear.

Most of the lipids absorbed by the intestine are transferred to the blood through the thoracic duct (Fraser et al., 1968), and it is not clear if lipid absorption would be affected by the ligation of the thoracic duct. Therefore, we performed a prospective, quasirandom, case-control study, in the hope of finding out the change in lipid metabolism after esophagectomy.

\section{MATERIAL AND METHODS}

\section{Study population}

From January 2008 to December 2009, 305 patients were definitively diagnosed with middle or upper esophageal carcinoma after esophagectomy, and they were assigned to the thoracic duct ligation group and the quasi-randomly controlled group. Finally, 74 medical cases of esophageal carcinoma were included, among which 54 were males and 20 females, with age ranging from 34 to 74 years. There were 39 cases in the thoracic duct ligation group and 35 other cases in the group without ligation. Their general information is given in Table 1. 


\section{Assignment}

The patients with esophageal carcinoma were continuously prospectively divided into the thoracic duct ligation group and the quasi-randomly controlled group by checking the oddeven hospital number. The patients with odds numbers assigned to the ligation group while the others in the non-ligated group. Eligible patients were contacted by the study researcher who obtained baseline data and informed consent. Patients were told that the study would compare two types of operation quasi-randomly; routine esophagectomy (control group) or routine esophagectomy plus thoracic duct ligation (intervention), and the risk of thoracic duct ligation or not. The patients agreed with this study, signed the informed consent form and were then selected as the second step. The ethical standards of the Helsinki Declaration of 1975 were followed. Third, patients were excluded for the following reasons: they had metabolic disorders, such as lipoprotein disorder, diabetes mellitus, hyperthyroidism, etc.; they suffered infection, chylothorax, fistula and stenosis of anastomotic stoma, or failure of enteral nutrition; or they missed appointments. Finally, a total 74 patients satisfied the inclusion criteria and were analyzed.

\section{Interventions}

Radical esophagectomy, clearance of regional lymph nodes and esophageal-gastric anastomosis were performed in all patients. In the ligation group, the posterior mediastinum was exposed before full disassociation of the stomach and esophagus, as well as the esophageal-gastric anastomosis during the operation. Between the planum of the inferior pulmonary vein (at the T8 level) and $3-5 \mathrm{~cm}$ above the diaphragm, an incision of about $1.0 \mathrm{~cm}$ of the mediastinal pleura was made on the right side of the thoracic aorta, and the tissues between the aorta and the azygos vein were separated in bundles from the thoracic aorta near the thoracic spine by small right-angle forceps. Finally these bundles of tissues including the thoracic duct were ligated together, and the ligation was not too tight nor too loose. For several tissue bundles separated unsatisfactorily, prevertebral tissues including azygos vein could be ligated in bulk to avoid missing the ligation of the thoracic duct. After ligation, the chylous exudates and hemorrhage of azygos vein ware carefully examined at the ligation site and its lower part.

\section{Nutritional support}

A nasopharynx-duodenum tube was used in all patients in both groups during the operation. Enteral nutritional support was performed starting on the 1st day after surgery and lasted until the 8th day. Patients were placed on a liquid diet starting on the 6th day after surgery, and the quantity was increased gradually. The patients were discharged between the 10th and 14th day. Enteral nutrient emulsion (Fresubin, Sino-Swed Pharmaceutical Corp. Ltd.) was administered through the nasopharynx feeding tube, and $500 \mathrm{~mL}$ emulsion was given on the 1st postoperative day. Afterwards, $1000-1500 \mathrm{~mL}$ emulsion was used starting on the 2nd day ( $1000 \mathrm{~mL}$ emulsion given to patients with body weight $\leq 50 \mathrm{~kg}$ ). Semi-liquid food was added at a normal quantity starting on the 9th day after surgery, and normal food was started on the 14th day after surgery. Before the operation, nutritional support was not provided to the patients in either group, and no blood, blood products or fat emulsions were infused during or after the surgery. During the study, the patients who received blood, blood plasma, proteins or fat emulsions, whose nutrition tube fell off and changed to abenteric nutrient support during or 
after surgery, and who suffered digestive disorders were all excluded from this study.

\section{Data collecting and processing}

A fasting venous blood sample of $5 \mathrm{~mL}$ was collected in the morning on the 2nd day after hospitalization, the 9th day after surgery, and the end of the 1st month and 3rd month after surgery. The concentrations of cholesterol (CHOL), triglycerides (TG), high-density lipoprotein (HDL), and low-density lipoprotein (LDL) in plasma were analyzed by HITACHI Modular SWA P/E automatic biochemistry and immune analysis equipment. All data are reported as means \pm standard deviation and statistically analyzed by the SPSS16.0 software. The blood lipid variation curves in both groups were compared by repeated measurement, and the mean $t$-test value was used for all data comparisons between the two groups.

\section{RESULTS}

No patient died in either group. The baseline between the ligation group and the nonligation group was equal (Table 1). There were no significant differences regarding gender, age, volume of enteral nutrient taken daily, total amount of thoracic fluid, and diarrhea between the two groups. Therefore, it was feasible to compare the plasma lipid levels. The plasma concentrations of CHOL, TG, HDL, and LDL are compared in Table 2.

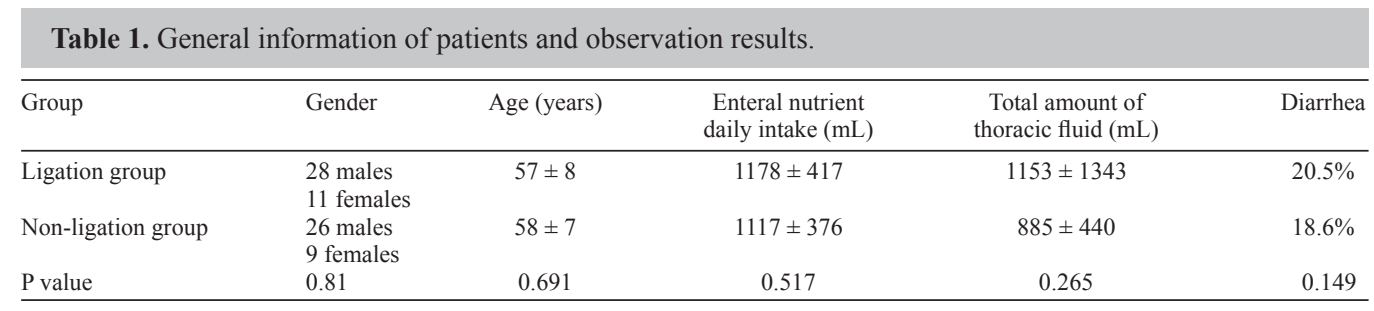

Table 2. Results of biochemical analysis in blood lipids.

\begin{tabular}{llcccc}
\hline & & $\begin{array}{c}\text { Before surgery } \\
(\mathrm{mM})\end{array}$ & $\begin{array}{c}\text { 9th day after surgery } \\
(\mathrm{mM})\end{array}$ & $\begin{array}{c}\text { 1st month after surgery } \\
(\mathrm{mM})\end{array}$ & $\begin{array}{c}\text { 3rd month after surgery } \\
(\mathrm{mM})\end{array}$ \\
\hline $\mathrm{CHOL}$ & Ligation group & $4.16 \pm 0.86$ & $3.52 \pm 0.73$ & $3.75 \pm 0.69$ & $3.94 \pm 0.42$ \\
$(\mathrm{mM})$ & Non-ligation group & $4.44 \pm 1.17$ & $3.81 \pm 0.62$ & $4.05 \pm 0.66$ & $3.68 \pm 0.77$ \\
& P value & 0.238 & 0.071 & 0.058 & 0.074 \\
$\mathrm{TG}$ & Ligation group & $1.18 \pm 0.84$ & $1.02 \pm 0.43$ & $1.07 \pm 0.39$ & $1.52 \pm 0.47$ \\
$(\mathrm{mM})$ & Non-ligation group & $0.96 \pm 0.39$ & $1.08 \pm 0.37$ & $1.20 \pm 0.34$ & $1.37 \pm 0.38$ \\
& P value & 0.135 & 0.539 & 0.143 & 0.083 \\
$\mathrm{HDL}$ & Ligation group & $1.33 \pm 0.45$ & $1.12 \pm 0.39$ & $1.30 \pm 0.36$ & $1.28 \pm 0.22$ \\
$(\mathrm{mM})$ & Non-ligation group & $1.69 \pm 1.13$ & $1.10 \pm 0.40$ & $1.19 \pm 0.29$ & $1.33 \pm 0.47$ \\
& P value & 0.081 & 0.875 & 0.146 & 0.176 \\
LDL & Ligation group & $2.80 \pm 1.02$ & $2.11 \pm 0.89$ & $1.82 \pm 0.65$ & $2.01 \pm 0.61$ \\
$(\mathrm{mM})$ & Non-ligation group & $2.86 \pm 1.00$ & $2.53 \pm 0.63$ & $2.54 \pm 0.63$ & 0.000 \\
& P value & 0.794 & 0.025 & & $2.50 \pm 0.67$ \\
& & & & 0.002 \\
\hline
\end{tabular}

The time-dependent variation in blood lipids determined by whole blood biochemical analysis are shown in Figures 1-4. There were no significant statistical differences in CHOL, TG and HDL concentration between the two groups before and after surgery at various time points. However, LDL concentration in the ligation group on the 9th day after surgery was sig- 
nificantly different from that observed before surgery and in the non-ligation group, where it reached the lowest point at the end of the 1st month and then gradually recovered after the 3rd postoperative month, but it was still lower than in the non-ligation group and before surgery.

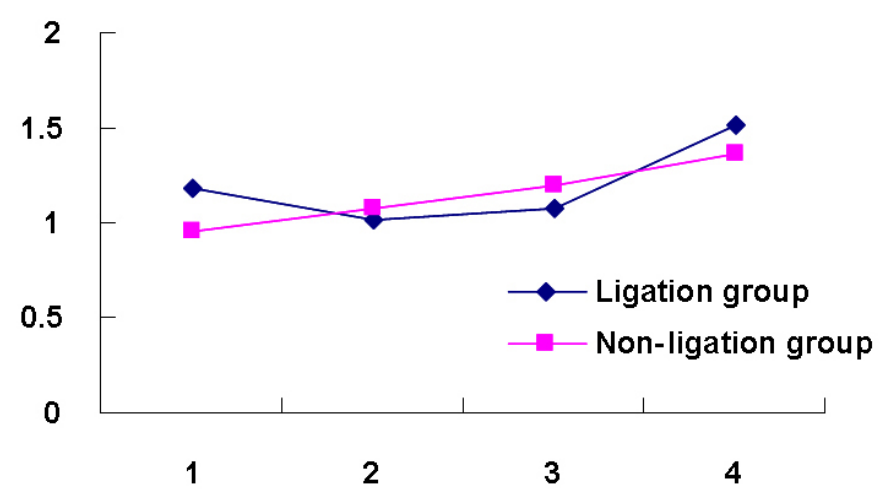

Figure 1. Variation of TG in the two groups (P value 0.897).

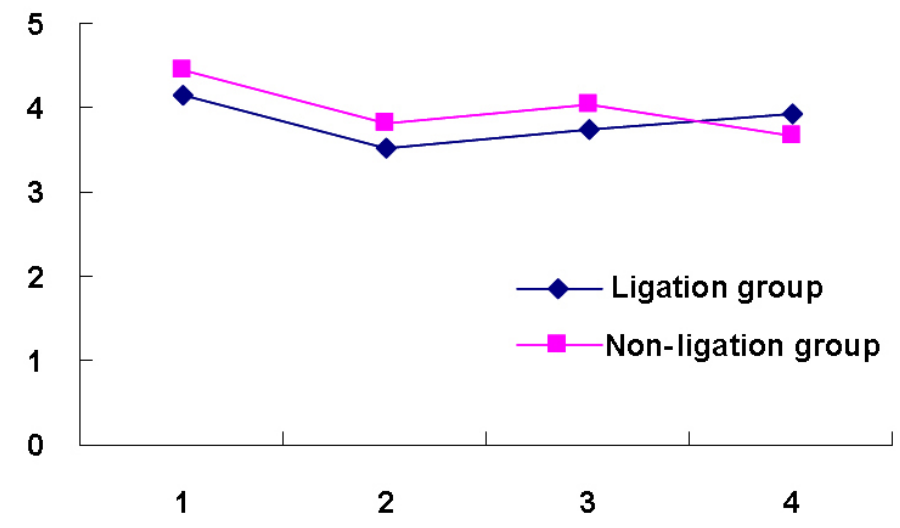

Figure 2. Variation of CHOL in the two groups (P value 0.911).

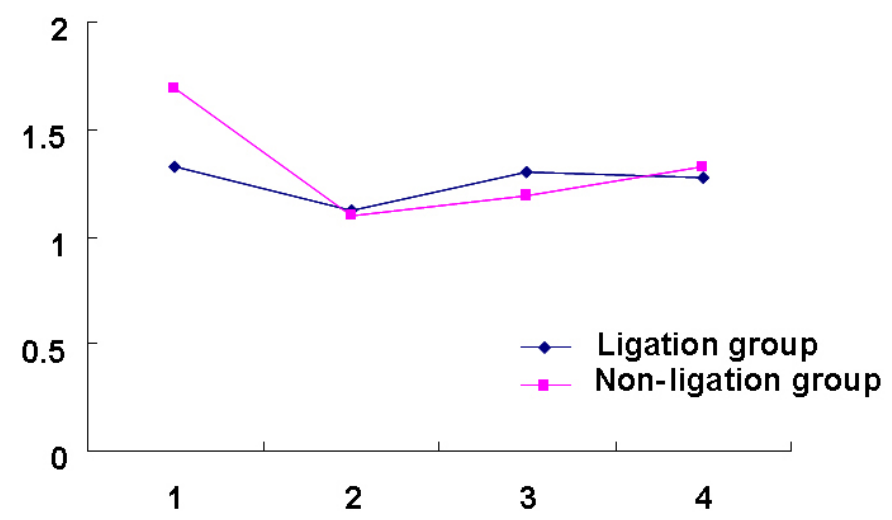

Figure 3. Variation of HDL in the two groups (P value 0.450 ). 


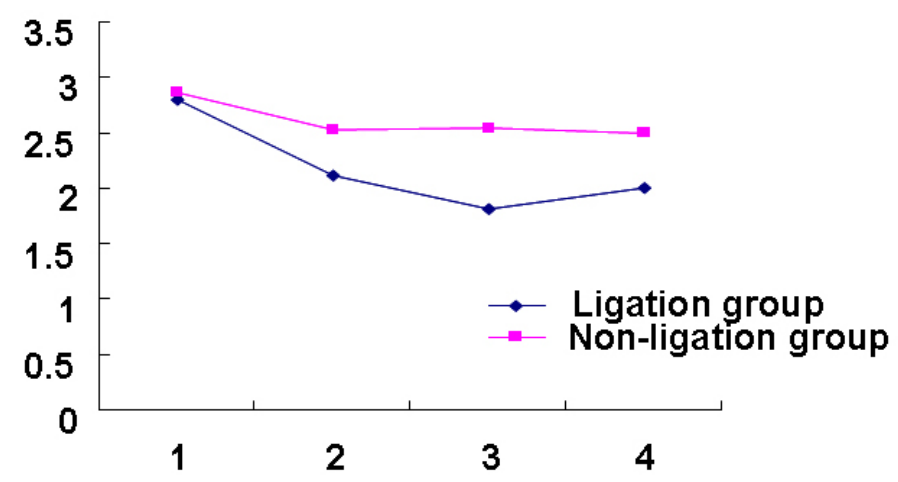

Figure 4. Variation of LDL in the two groups (P value 0.000).

\section{DISCUSSION}

Postoperative chylothorax is the most severe complication associated with esophagectomy. Chylothorax refers to the presence of lymphatic fluid in one or both pleural spaces, secondary to leakage in the thoracic duct or one of its main tributaries (Miller, 2005), where risk of death is high without treatment (Fahimi et al., 2001). Prophylactic thoracic duct ligation is still an effective procedure to reduce the incidence of chylothorax (Sokouti and Montazeri, 2009).

The anatomic basis of prophylactic thoracic duct ligation is as follows: thoracic duct arises from cisterna chyli at the lower edge of the 12th vertebra, then ascending into the chest through the aortic foramen, and afterwards going through the space between the thoracic aorta and azygos vein at the height of the 5th thoracic vertebra and next sloping towards the left through the interspace between the esophagus and vertebrae. It then moves upward through the interspace between the esophagus and mediastinal pleura to the neck, finally entering the venous system. There are many variations of the thoracic duct, and the collateral circulation is abundant (Hematti and Mehran, 2011). It is generally considered that the thoracic duct at 5-6 $\mathrm{cm}$ above the diaphragm is usually single, and therefore, to make sure no chylothorax occurred after thoracic duct ligation, all thoracic ducts were ligated at this part in the ligation group.

The main physiological function of the thoracic duct is to transfer the digested lipid to the venous system (Arunabh and Fein, 1997; Lee et al., 2008; Seeger et al., 2009). The dietary lipids consist mainly of fat, along with a small amount of phospholipids, cholesterol, etc. The lipids are mainly digested in the upper part of small intestine, and the digestive products are mainly absorbed in the lower part of the duodenum and the upper part of jejunum. Among them, triglycerides, which are formed by middle-chain fatty acids (6-10C) and short-chain fatty acids (2-4C) are hydrolyzed into fat acids and glycerin under the action of lipases in intestinal mucosal cell, then enter the blood circulation by the portal vein. Triglycerides are synthesized from long-chain fatty acids (12-26C) and 2-monoglyceride by the catalysis of acetyl-coenzyme A transferase in the smooth endoplasmic reticulum (monoglyceride pathway), and then bind to apolipoproteins (apo), such as apoB48, apoC, apoA-I, apoA-IV, etc., as well as phospholipids and cholesterol to form the chylomicrons (CM). The triglycerides then enter the chyliferous vessel on the epithelial villi of intestinal mucosa and are finally transferred to the blood circulation through "left and right intestinal trunks $\rightarrow$ cisterna chyli $\rightarrow$ thoracic duct" pathway (Light and Lee, 2010). It turned out that there was no influence of thoracic duct liga- 
tion on the absorption of triglycerides formed by middle-chain and short-chain fatty acids. However, it could affect the absorption of triglycerides synthesized in the "monoglyceride pathway". Therefore, important questions arise. Is there any change in the transportation, absorption and metabolism of lipids in vivo after acute blocking of the thoracic duct in esophageal carcinoma surgery? For collateral lymphatic circulation, when could it be well established, and when could it be competent enough for the normal working of the thoracic duct? A few studies were found by reviewing articles.

Therefore, we compared the levels of CHOL, TG, HDL, and LDL in fasting blood samples in the ligation group and the non-ligation group to answer these questions.

All lipids contained in blood are called blood lipids, which composition is complex and includes triglycerides, phospholipids, cholesterol, and free fatty acids. They come from exogenous lipids (lipids absorbed into blood from foods), and endogenous lipids (synthesized by liver, lipid cells and other tissues) that have been released into the blood. Blood lipids do not dissolve in water, and they are transferred in the form of lipoprotein after binding with apolipoprotein. By differences in density, lipoprotein can be divided into CM, very low-density lipoprotein (VLDL), LDL, and HDL (Mahley et al., 2007; Semenkovich, 2007).

Liver, adipose tissue and small intestine are the main sites of triglyceride synthesis, and the synthetic capability of liver is the strongest. Triglycerides are synthesized in the endoplasmic reticulum and bind with phospholipids and cholesterol to form VLDL, which is then transferred out of the liver through the blood. Glycerin and fatty acids that are needed in the synthesis of triglycerides are provided by glucose metabolism, and even if there is no fat ingested by humans or animals, a great amount of CHOL can be synthesized from sugar. When fasting, hunger of excitement of the sympathetic nerve occurs, the secretions of epinephrine, norepinephrine and glucagon increase to activate the lipases to decompose the reserved fat into glycerin and fatty acids (Klein and Romijn, 2007). A great amount of energy released through $\beta$-oxidation of fatty acids is provided to be utilized by the organisms in the form of ATP (Fry, 2010). It could be seen, from the metabolic process of TG, that there was no influence on its synthesis and decomposition by thoracic duct ligation. It was also found in this study that when we compared the data between the thoracic duct ligation group and the non-ligation group at 4 different time points, there was no difference in concentrations of TG between them (before surgery, $\mathrm{P}=0.135 ; 1$ st week after surgery, $\mathrm{P}=0.539 ; 1$ st month after surgery, $\mathrm{P}=0.143$; and $3 \mathrm{rd}$ month after surgery, $\mathrm{P}=0.083)$. Moreover, there was also no difference in the variation curves of the TG concentration between the two groups $(\mathrm{P}=0.897)$.

Cholesterol can be synthesized in almost all tissues in the whole organism except the brain tissue in adult animals and the mature erythrocyte, and liver is the main site of cholesterol synthesis. Acetyl-COA is the material of steroid acid synthesis that is produced by the catabolism of glucose, amino acids and fatty acids in the mitochondrion. Cholesterol can be converted into cholic acid in vivo and released into intestine with bile. It can be converted into steroid hormones. It can be oxidized into 7-dehydrocholesterol and then transformed into vitamin D3 under UV irradiation. It could be seen that CHOL metabolism was also not affected by thoracic duct ligation. It was also found in this study that when we compared the results between the thoracic duct ligation group and the non-ligation group at 4 time points, there was no significant difference in concentrations of CHOL between the two groups (before surgery, $\mathrm{P}=0.238 ; 1$ st week after surgery, $\mathrm{P}=0.071 ; 1$ st month after surgery, $\mathrm{P}=0.058$; 3rd month after surgery, $\mathrm{P}=0.074)$. Furthermore, there was also no significant difference in the variation curves of CHOL concentration between the two groups $(\mathrm{P}=0.911)$. 
HDL is mainly synthesized by the liver and small intestine. ApoA-I as well as phospholipid and cholesterol, which break off CM and VLDL, form the new HDL. It can be seen that HDL in blood can be endogenously synthesized when absorption of exogenous fat is hindered. In this study, it was also found that when we compared the values between the thoracic duct ligation group and the non-ligation group at 4 time points, there was no significant difference in HDL concentrations between the two groups (before surgery, $\mathrm{P}=0.081$; 1 st week after surgery, $\mathrm{P}$ $=0.875 ; 1$ st month after surgery, $\mathrm{P}=0.146 ; 3$ rd month after surgery, $\mathrm{P}=0.176)$. Also, there was no difference in the variation curves of HDL concentration between the two groups $(\mathrm{P}=0.450)$.

$\mathrm{CM}$ is the main pattern of transportation of exogenous triglycerides and cholesterol. Fat is digested and absorbed, and triglycerides can be synthesized by intestinal mucosal cells, then transferred to blood with phospholipid and cholesterol, which are synthesized and absorbed, as well as CM, which is newly generated from apolipoproteins such as (apo) A-I, A-II, A-IV and B48 through lymphatic vessels. Mature CM are then generated by exchanging apo with HDL and are decomposed and utilized by lipoprotein lipases on the surfaces of capillary endothelial cells in different tissues. Its metabolism in plasma is rapid, of which the half-life is 5 to $15 \mathrm{~min}$, and CM are free in the empty stomach (Pláteník, 2011). Fasting blood samples collected in the morning were used in the biochemical analysis in this study, but CM was not considered in the analysis.

LDL is synthesized by liver, which is the main pattern of endogenous cholesterol transportation, and LDL is transformed from VLDL. It is ingested and decomposed by tissues such as liver, adrenal cortex, ovary, and testis. The half-life of LDL in vivo is 2-4 days. As mentioned above, triglycerides and cholesterol generated in the monoglyceride pathway need to be bound to apoproteins (belonging to $\beta$-lipoprotein) synthesized by intestinal epithelial cells to form $\mathrm{CM}$ and transferred to the blood through the thoracic duct. When apoproteins cannot be sufficiently synthesized by intestinal epithelial cells, CM cannot be generated or transferred out of cells into the blood, resulting in the lack of $\beta$-lipoprotein in the blood. $\beta$-lipoprotein is LDL (Rhoades and Bell, 2009; Pláteník, 2011). Therefore, thoracic duct ligation can lead to the lack of LDL in the blood. In this study, the LDL levels in the ligation group were clearly lower than those in the non-ligation group at various time points, and they were lowest in the 1st month and gradually recovered by the $3 \mathrm{rd}$ postoperative month. It indicated that the transportation of CM into the blood was blocked by thoracic duct ligation, which hindered the absorption of fat and exerted unfavorable effects on fat metabolism in vivo and enteral nutrition support. It also indicated that the establishment of collateral circulation after thoracic duct ligation was slower, which had negative effects on the early nutrition of the esophageal carcinoma patients after surgery.

In this study, we showed that there were no effects of thoracic duct ligation on the plasma concentrations of TG, CHOL and HDL, but that ligation did affect the concentration of LDL, which was the lowest in the 1st month after surgery. Because the physiological function of LDL is to transfer cholesterol from liver to tissues, a decrease in plasma LDL concentration hinders cholesterol transportation and furthermore results in the lack of cholesterol in tissues, leading to corresponding physiological functional disorders. Early enteral nutrition along with parenteral lipids have been recommended for the ligated group, while only an enteral nutrition schedule for the non-ligated group, to enhance recovery.

Pathological and physiological changes after thoracic duct ligation still need to be examined by further experiments, including lymphangiography, route of metastasis of tumor cells. However, the results of this study illustrated that the thoracic duct ligation is effective in preventing chylothorax after esophageal carcinoma surgery, which has been clinically used for 
many years and can result in unfavorable clinical consequences. In the esophageal carcinoma dissection operation, prophylactic thoracic duct ligation is not recommended for patients without obvious damage to the thoracic duct.

\section{Conflicts of interest}

The authors declare no conflict of interest.

\section{ACKNOWLEDGMENTS}

We thank the patients and caregivers who contributed to the study. We are greatly thankful to Yongjun Chen, PhD, Director of Suining Central Hospital, and Xianyu Sun, Vice Director of Suining Central Hospital, who provided much support for this study.

\section{REFERENCES}

Aiko S, Yoshizumi Y, Matsuyama T, Sugiura Y, et al. (2003). Influences of thoracic duct blockage on early enteral nutrition for patients who underwent esophageal cancer surgery. Jpn. J. Thorac. Cardiovasc. Surg. 51: 263-271.

Arunabh and Fein AM (1997). Chylothorax: A Review. Clin. Pulm. Med. 4: 63-70.

Cagol M, Ruol A, Castoro C, Alfieri R, et al. (2009). Prophylactic Thoracic Duct Mass Ligation Prevents Chylothorax After Transthoracic Esophagectomy for Cancer. World J. Surg. 33: 1684-1686.

Cerfolio RJ (2006). Chylothorax after esophagogastrectomy. Thorac. Surg. Clin. 16: 49-52.

Fahimi H, Casselman FP, Mariani MA, van Boven WJ, et al. (2001). Current management of postoperative chylothorax. Ann. Thorac. Surg. 71: 448-450.

Fraser R, Cliff WJ and Courtice FC (1968). The effect of dietary fat load on the size and composition of chylomicrons in thoracic duct lymph. Q. J. Exp. Physiol. Cogn. Med. Sci. 53: 390-398.

Fry M (2010). Essential Biochemistry for Medicine. Markono Print Media, Pte Ltd., Singapore, 22.

Hematti H and Mehran RJ (2011). Anatomy of the Thoracic Duct. Thorac. Surg. Clin. 21: 229-238.

Klein S and Romijn JA (2007). Chapter 35 - Obesity. In: Williams Textbook of Endocrinology, 11th edn. (Kronenberg HM, ed.). Saunders, Philadelphia, 1567-1569.

Lee H, Gumpeni R, Jain M and Talwar A (2008). MDChylothorax: a review of current management strategies: sequelae may include malnutrition and respiratory failure. J. Respir. Dis. 29: 325-333.

Light RW and Lee YCG (2010). Chapter 74 - Pneumothorax, Chylothorax, Hemothorax and Fibrothorax. In: Murray and Nadel's Textbook of Respiratory Medicine, 5th edn. (Mason RJ, ed.). Saunders, Philadelphia. Available at [http:// www.mdconsult.com/books/page.do?eid=4-u1.0-B978-1-4160-4710-0..00074-2-s0250\&isbn=78-1-4160-47100\&type=bookPage\&sectionEid=4-u1.0-B978-1-4160-4710-0..00074-2-s0250\&uniqId=239149877-4]. $\quad$ Accessed April 12, 2011.

Mahley RW, Weisgraber KH and Bersot TP (2007). Lipid Biochemistry and Cholesterol Metabolism. In: Williams Textbook of Endocrinology. 11th edn. (Kronenberg HM, ed.). Saunders, Philadelphia, 1589-1593.

Miller JI (2005). Anatomy of the Thoracic duct and Chylothorax. In: General Thoracic Surgery. 6th edn. (Shields TW, ed.). Lippincott William \& Wilkins Co., Philadelphia, 879-888.

Nair SK, Petko M and Hayward MP (2007). Aetiology and management of chylothorax in adults. Eur. J. Cardiothorac. Surg. 32: 362-369.

Pláteník J (2011). Lipoproteins Biochemical examination of lipid metabolism. Available at [http://che1.lf1.cuni.cz/ html/05_Lipoproteins_theory_v2.pdf]. Accessed April 6, 2011.

Rhoades RA and Bell DR (2009). Medical physiology: principles for clinical medicine. 3rd edn. Lippincott Williams \& Wilkins, Philadelphia, 463-543.

Sauvanet A (2002). Surgery technique preventive ligation of the thoracic duct during esophagectomy for cancer. Ann Chir 127: 228-321.

Seeger M, Bewig B, Günther R, Schafmayer C, et al. (2009). Terminal part of thoracic duct: high-resolution US imaging. Radiology 252: 897-904.

Semenkovich CF (2007). Chapter 217 - Disorders of lipid metabolism. In: Cecil Medicine, 23rd edn. (Goldman L, 
ed.). Saunders, Philadelphia, Available at http://www.mdconsult.com/books/page.do? eid=4-u1.0-B978-1-41602805-5..50222-6\&isbn=978-1-4160-2805-5\&type=bookPage\&sectionEid=4-u1.0-B978-1-4160-2805-5..502226\&uniqId=239149877-8\#4-u1.0-B978-1-4160-2805-5..50222-6. Accessed April 12, 2011.

Sokouti M and Montazeri V (2009). Results of prophylactic thoracic duct ligation in advanced esophageal carcinoma. Ind. J. Thorac. Cardiovasc. Surg. 25: 18-22. 